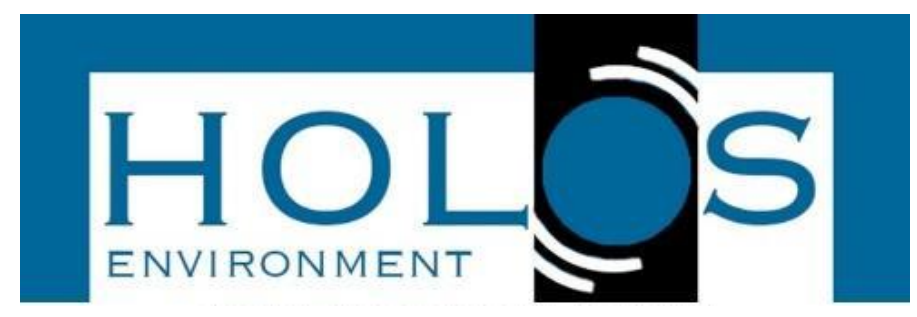

\title{
BAIRROS DE CLASSES ECONÔMICAS DISTINTAS APRESENTAM IGUAIS INDICADORES DE SALUBRIDADE AMBIENTAL? ESTUDO DE CASO: PATOS DE MINAS-MG
}

\author{
EVALUATION OF THE ENVIRONMENTAL HEALTH INDICATOR IN \\ NEIGHBORHOODS OF DIFFERENT ECONOMIC CLASSES
}

\author{
Mariana de Fatima Soares ${ }^{1}$; Frederico Carlos Martins de Menezes Filho ${ }^{1}$
}

Artigo recebido em: 29/07/2020 e aceito para publicação em: 07/10/2020.

DOI: http:/dx.doi.org/10.14295/holos.v21i1.12412

\begin{abstract}
Resumo: $\mathrm{O}$ acesso ao saneamento ambiental possibilita qualidade de vida e dignidade ao ser humano. Nesse sentido, o presente trabalho faz uma avaliação da relação entre a renda média mensal e a salubridade ambiental através da aplicação do Indicador de Salubridade Ambiental (ISA) em bairros de distintas classes econômicas na cidade de Patos de Minas-MG. Para isso, foram utilizados dados do Instituto Brasileiro de Geografia e Estatística (IBGE), da Prefeitura Municipal e da companhia de saneamento para o cálculo dos Indicadores de Abastecimento de Água, Esgoto Sanitário, Resíduos Sólidos, Drenagem Urbana, Controle de Vetores e Socioeconômico. Dos oito bairros analisados, apenas um apresentou média salubridade, enquanto os demais baixa salubridade. O Indicador Socioeconômico foi o mais decisivo para tal classificação. Em paralelo, destaca-se a nulidade para o Indicador de Drenagem Urbana, evidenciando a necessidade de ações prioritárias para implantação de novas estruturas e manutenção das existentes. Conclui-se que o acesso ao saneamento ambiental diverge entre os bairros em função da renda média contrapondo à universalização tão desejada por todos e preconizada pela lei do saneamento.
\end{abstract}

Palavras-chave: Saneamento ambiental. ISA. Indicador Socioeconômico.

Abstract: Access to environmental sanitation enables quality of life and human dignity. This study evaluates the association between average monthly income and environmental health through the use of Environmental Health Indicator (EHI) in neighborhoods of different economic classes in the municipality of Patos de Minas-MG. For this, data from the Brazilian Institute of Geography and Statistics (IBGE), the City Hall and the sanitation company were used to calculate the Indicators of Water Supply, Sanitary Sewage systems, Solid Waste, Urban Drainage, Vector Control and Socioeconomic. Of the eight neighborhoods analyzed, only one presented medium healthiness, while the other low healthiness. The Socioeconomic Indicator was the most decisive for this classification. On the other hand, the nullity for the Urban Drainage Indicator stands out, evidencing the need for priority actions for the implementation of new structures and maintenance of existing ones. We concluded that the access to environmental sanitation diverges among the neighborhoods according to the average income, as opposed to the universalization so desired by all and recommended by the sanitation law.

Keywords: Environmental sanitation. EHI. Socioeconomic Indicator. 


\section{INTRODUÇÃO}

Com intuito de garantir a qualidade de vida do ser humano, a Organização das Nações Unidas, em 2010 (ONU, 2015), reconheceu o direito ao saneamento básico, além do direito à saúde, à vida e à educação. As consequências do déficit do saneamento impactam negativamente tanto a dignidade humana quanto a saúde física e mental (BROWN; NEVES-SILVA; HELLER, 2016).

No Brasil, a Lei no 11445 conhecida como a Lei do Saneamento, discorre sobre o conjunto de serviços que devem ser disponibilizados para a sociedade (BRASIL, 2007). São eles: o abastecimento de água potável, o esgotamento sanitário, a limpeza urbana, o manejo de resíduos sólidos e, por último a drenagem e o manejo das águas pluviais urbanas. O Instituto Trata Brasil (2020) destaca que a realidade do país não é satisfatória, visto que cerca de 35 milhões de brasileiros não têm acesso à água tratada e 100 milhões são desassistidos pela coleta de esgoto domiciliar. Além disso, evidencia-se que no país, apenas $43 \%$ do esgoto é coletado e tratado e $27 \%$ sem coleta, nem tratamento.

Acerca da garantia da qualidade de água, Heller (2006) discute sobre a caracterização de suas impurezas físicas, químicas e biológicas. Lisboa, Heller e Silveira (2013) afirmam que "a indisponibilidade de recursos financeiros e a limitação quanto à qualificação profissional e capacidade técnica municipal" são os principais gargalos enfrentados para averiguar a água de forma confiável.

Por outro lado, quanto ao manejo de águas pluviais, o diagnóstico do Sistema Nacional de Informações sobre Saneamento (SNIS) mostrou a evolução deste componente, com a redução percentual de alagamentos de $43,5 \%$ para $32,9 \%$ no período de 2008 a 2018 (SNIS, 2018). Tal melhoria fora acompanhada de um investimento na região sudeste de 2,49 milhões de reais impactando na redução de $20 \%$ dos alagamentos existentes (SNIS, 2018).

Segundo Uhr et al. (2016), a proteção contra doenças de veiculação hídrica está diretamente relacionada à presença de rede coletora de esgotos local e à qualidade da água distribuída. Salla et al. (2019) relatam que investimentos no setor de saneamento, como a coleta e o devido destino final do esgoto, são de suma importância e incidem diretamente nos gastos com saúde. Ainda, segundo os autores, em termos numéricos, a cada dólar investido em saneamento há uma redução de, 
aproximadamente, 4,23 dólares no custo com recursos médicos. Por isso, deve-se buscar um planejamento que contenha o investimento na saúde e também em infraestrutura de redes de esgoto e abastecimento, no intuito de garantir resultados positivos de natureza social e ambiental. A falta de saneamento acarreta a proliferação de doenças, que podem levar a óbito, ou ao afastamento do emprego e da escola, devido à falta de condições físicas adequadas para manter as atividades normalmente desenvolvidas (TRIGUEIRO et al., 2016).

Em relação aos resíduos sólidos, a Associação Brasileira de Empresas de Limpeza Pública e Resíduos Especiais (ABRELPE, 2019) constatou que o Brasil, em 2018, produziu cerca de 79 milhões de toneladas de resíduos sólidos e aumentou $1 \%$ a sua produção em comparação ao ano de 2017. Entretanto, apesar de $92 \%$ desse montante terem sido coletados, apenas 59,5\% apresentaram destino adequado em aterros sanitários. Logo, apesar da evolução na coleta de lixo, o descarte correto é fundamental para manter o equilíbrio ambiental. Santaella et al. (2014) destacam que o destino final correto dos resíduos sólidos é garantido por meio da reutilização, reciclagem, compostagem, aproveitamento da energia disponibilizada e a disposição em aterros sanitários. Só assim se poderá fornecer alternativas para questões climáticas, poluição atmosférica e perda da biodiversidade (CADERNO DE GRADUAÇÃO, 2015).

Nesse contexto, equacionar e demonstrar em números a real situação de cada país, cidade, bairro ou até mesmo uma comunidade, torna-se extremamente necessário. De fato, evidenciar o quão preocupante é a situação mundial é relevante, pois muitas vezes a população não está atenta aos riscos e as consequências presentes e futuras. Em 1999, a fim de mensurar os níveis de salubridade ambiental, foi criado o Índice de Salubridade Ambiental (ISA), pelo Conselho Estadual de Saneamento (CONESAN) de São Paulo (CONESAN, 1999). Através do cálculo do ISA, é possível categorizar o local de estudo como insalubre, baixa salubridade, média salubridade ou ótima salubridade.

Segundo Teixeira, Filho e Santiago (2018), desde a criação do ISA até o ano de 2016, foram realizados 60 estudos em todo o Brasil. A região sul detinha o maior número em todo o país, com 20 estudos, e a região norte possui a menor quantidade, com apenas um estudo. 
Menezes Filho e Rodrigues (2015) desenvolveram o ISA para a cidade de Rio Paranaíba-MG utilizando dados referentes ao abastecimento de água, esgoto sanitário, resíduos urbanos e drenagem urbana. Com base na pesquisa, puderam classificar a cidade como de baixa salubridade, principalmente, devido ao déficit de dispositivos de microdrenagem.

Teixeira (2017) calculou o ISA para a cidade Ouro Preto-MG, utilizando os dados de abastecimento de água, esgoto sanitário, resíduos urbanos, drenagem urbana, controle de vetores, socioeconômicos e recursos hídricos, e constatou que a classificação de baixa salubridade, possivelmente era devido a fatores de maior influência, como o esgoto sanitário e resíduos urbanos.

Diante disso, o presente artigo propõe avaliar a salubridade ambiental de bairros com distintas rendas econômicas na cidade de Patos de Minas-MG, utilizandose da metodologia ISA proposta por CONESAN (1999) e adaptada por diversos autores (OLIVEIRA, 2014; LINS; MORAES, 2017; LIMA; ARRUDA; SCALIZE, 2019).

\section{MATERIAL E MÉTODOS}

A cidade de Patos de Minas está situada na região do Alto Paranaíba em Minas Gerais, nas coordenadas: latitude $18^{\circ} 22^{\prime} 2^{\prime \prime}$ 'S e longitude $46^{\circ} 54^{\prime} 54^{\prime \prime} \mathrm{O}$. Localiza-se na região de planalto central, ou seja, possui áreas altas e planas entre serras e vales, com altitude média de $900 \mathrm{~m}$. Além disso, sua precipitação média anual é de 1.499 $\mathrm{mm}$, temperatura média anual de $22,8^{\circ} \mathrm{C}$ (SILVA, 2015) e no último censo do Instituto Brasileiro de Geografia e Estatística (IBGE) apresentava uma população de 138.836 habitantes (IBGE, 2012).

Os bairros a serem analisados foram definidos de acordo com a renda média mensal, utilizando-se dados do IBGE (2012). Selecionaram-se, então, os bairros Alto dos Caiçaras e Cônego Getúlio, considerados de alta renda; os bairros Rosário, Cerrado, Cristo Redentor e Nossa Senhora das Graças, de renda intermediária e os bairros Alto da Colina e Jardim Esperança, de baixa renda.

\subsection{Obtenção de dados sobre os bairros de estudo}

Consultou-se diferentes fontes e dados para o desenvolvimento desse trabalho (Tabela 1). Nessa etapa, procedeu-se também ao levantamento de campo para ava- 
liação de possíveis focos de resíduos e erosões decorrentes da falta de drenagem urbana nos bairros em estudo.

Tabela 1 - Dados e fontes consultados para elaboração do trabalho

Tipo de dados

Indicador de Cobertura de Abastecimento de Água ( $\left.\mathrm{I}_{\mathrm{CA}}\right)$, Indicador de Cobertura em Coleta de Esgoto $\left(\mathrm{I}_{\mathrm{CE}}\right)$, Indicador de Esgoto Tratado $\left(\mathrm{I}_{\mathrm{TE}}\right)$, Renda média dos bairros analisados.

Indicador de Qualidade da Água Distribuída $\left(\mathrm{l}_{\mathrm{QA}}\right)$.

Frequência da Coleta; Condição atual da Coleta seletiva, Reciclagem e Aterro; Número de casos de Dengue, Esquistossomose, Leptospirose; Mapeamento da infraestrutura de drenagem.

Fonte: Elaborado pelos autores.

\subsection{Indicador de salubridade ambiental (ISA)}

O ISA foi criado para propor diretrizes no intuito de subsidiar a aplicação de recursos e facilitar a identificação das demandas para melhoria dos serviços em saneamento ambiental. Com o propósito de obter o resultado final do ISA, calcula-se a média ponderada de Indicadores específicos e relacionados, direta ou indiretamente, com a salubridade ambiental.

De acordo com Teixeira (2018), são empregados diferentes pesos, pois cada local possui realidades diferentes. Assim, para o referido trabalho, propôs-se a Equação 1:

$I S A=0,20 I_{A B}+0,20 I_{E S}+0,20 I_{R S}+0,20 I_{D U}+0,10 I_{S E}+0,10 I_{C V}$

Deste modo, classifica-se a situação de salubridade ambiental, em função da pontuação calculada para o ISA conforme a Tabela 2.

Tabela 2 - Nível de salubridade ambiental em função da faixa de pontuação do ISA.

\begin{tabular}{ll}
\hline Situação de Salubridade & Pontuação \\
\hline Insalubre & $0-0,25$ \\
Baixa Salubridade & $0,26-0,50$ \\
Média Salubridade & $0,51-0,75$ \\
Salubre & $0,76-1,00$
\end{tabular}

Fonte: Adaptado de DIAS (2003). 
O Indicador de Abastecimento de Água é o resultado da média aritmética entre o Indicador de Cobertura de Abastecimento de Água e o Indicador de Qualidade da Água Distribuída, expresso pela Equação 2.

$I_{A B}=\frac{I_{C A}+I_{Q A}}{2}$

Em que: $I_{C A}$ - Indicador de Cobertura de Abastecimento de Água; $I_{\mathrm{QA}}$ Indicador de Qualidade da Água Distribuída; $I_{A B}$ - Indicador de Abastecimento de Água.

O Indicador de Cobertura de Abastecimento de Água $\left(\mathrm{I}_{\mathrm{CA}}\right)$ possui por definição do CONESAN (1999) a razão entre o número de domicílios urbanos atendidos e o total de domicílios urbanos, expressa pela Equação 3.

$\mathrm{I}_{\mathrm{CA}}=\frac{\mathrm{D}_{\mathrm{UA}}}{\mathrm{D}_{\mathrm{UT}}}$

Em que: $D_{U A}$ - Domicílios urbanos atendidos; $D_{U T}$ - Domicílios urbanos totais; $\mathrm{I}_{\mathrm{CA}}$ - Indicador de Cobertura de Abastecimento de Água.

O Indicador de Qualidade da Água Distribuída $\left(I_{Q A}\right)$ é calculado pela Equação 4.

$\mathrm{I}_{\mathrm{QA}}=\mathrm{K}\left(\frac{\mathrm{N}_{\mathrm{AA}}}{\mathrm{N}_{\mathrm{AR}}}\right)$

Em que: $\mathrm{K}$ - Número de amostras realizadas; $\mathrm{N}_{\mathrm{AA}}$ - Quantidade de amostra considerada de água potável relativa à colimetria, ao cloro residual e à turbidez; $\mathrm{N}_{\mathrm{AR}}$ - Quantidade de amostras realizadas;

$\mathrm{I}_{\mathrm{QA}}$ - Indicador de Qualidade da Água Distribuída.

\subsubsection{Indicador de esgoto sanitário}

Segundo o CONESAN (1999) o Indicador de Esgoto Sanitário ( $\left.I_{E S}\right)$ é calculado 
pela média aritmética entre o Indicador de Cobertura em Coleta de Esgoto $\left(\mathrm{I}_{\mathrm{CE}}\right)$ e o Índice de Esgoto Tratado ( $\left.\mathrm{I}_{\mathrm{TE}}\right)$ (Equação 5).

$I_{E S}=\frac{I_{C E}+I_{T E}}{2}$

Em que: $I_{\mathrm{CE}}$ - Indicador de Cobertura em Coleta de Esgoto; $\mathrm{I}_{\mathrm{TE}}$ - Indicador de Esgoto Tratado; I IS - Indicador de Esgoto Sanitário.

O Indicador de Cobertura em Coleta de Esgoto é obtido pela Equação 6.

$\mathrm{I}_{\mathrm{CE}}=\frac{\mathrm{D}_{\mathrm{UE}}}{\mathrm{D}_{\mathrm{UT}}}$

Em que: $D_{U E}$ - Domicílios urbanos atendidos; $D_{U T}$ - Domicílios urbanos totais; $\mathrm{I}_{\mathrm{CE}}$ - Indicador de Cobertura em Coleta de Esgoto.

A pontuação final do $I_{C E}$ segue a Tabela 3 (CONESAN, 1999).

Tabela 3 - Pontuação final do Indicador de Cobertura em Coleta de Esgoto

\begin{tabular}{ccc}
\hline População urbana & Valor mínimo do $\mathrm{I}_{\mathrm{CE}}$ & Valor máximo do $\mathrm{I}_{\mathrm{CE}}$ \\
\hline Até 5 mil hab. & $\mathrm{I}_{\mathrm{CE}}<50 \% \rightarrow \mathrm{I}_{\mathrm{CE}}=0$ & $50 \%<\mathrm{I}_{\mathrm{CE}}<85 \% \rightarrow \mathrm{I}_{\mathrm{CE}}=1$ \\
De 5 a 20 mil hab. & $\mathrm{I}_{\mathrm{CE}}<55 \% \rightarrow \mathrm{I}_{\mathrm{CE}}=0$ & $55 \%<\mathrm{I}_{\mathrm{CE}}<85 \% \rightarrow \mathrm{I}_{\mathrm{CE}}=1$ \\
De 20 a 50 mil hab. & $\mathrm{I}_{\mathrm{CE}}<60 \% \rightarrow \mathrm{I}_{\mathrm{CE}}=0$ & $60 \%<\mathrm{I}_{\mathrm{CE}}<85 \% \rightarrow \mathrm{I}_{\mathrm{CE}}=1$ \\
De 50 a 100 mil hab. & $\mathrm{I}_{\mathrm{CE}}<65 \% \rightarrow \mathrm{I}_{\mathrm{CE}}=0$ & $65 \%<\mathrm{I}_{\mathrm{CE}}<85 \% \rightarrow \mathrm{I}_{\mathrm{CE}}=1$ \\
De 100 a 500 mil hab. & $\mathrm{I}_{\mathrm{CE}}<70 \% \rightarrow \mathrm{I}_{\mathrm{CE}}=0$ & $70 \%<\mathrm{I}_{\mathrm{CE}}<90 \% \rightarrow \mathrm{I}_{\mathrm{CE}}=1$ \\
$>500$ mil hab. & $\mathrm{I}_{\mathrm{CE}}<75 \% \rightarrow \mathrm{I}_{\mathrm{CE}}=0$ & $75 \%<\mathrm{I}_{\mathrm{CE}}<90 \% \rightarrow \mathrm{I}_{\mathrm{CE}}=1$ \\
\hline
\end{tabular}

Fonte: Adaptado CONESAN (1999).

O Indicador de Esgoto Tratado é obtido pela Equação 7.

$\mathrm{I}_{\mathrm{TE}}=\mathrm{I}_{\mathrm{CE}}\left(\frac{\mathrm{VT}}{\mathrm{VC}}\right)$

Em que: $I_{\mathrm{TE}}$ - Indicador de Esgoto Tratado; $\mathrm{I}_{\mathrm{CE}}$ - Indicador de Cobertura em Coleta de Esgoto; VT - Volume tratado de esgoto medido nas estações em áreas servidas por redes de esgoto; VC - Volume coletado de esgoto.

A pontuação final do $\mathrm{I}_{\mathrm{TE}}$ segue a Tabela 4 (CONESAN, 1999). 
Tabela 4 - Pontuação final do Indicador de Esgoto Tratado

\begin{tabular}{ccc}
\hline População urbana & Valor mínimo do $\mathrm{I}_{\mathrm{TE}}$ & Valor máximo do $\mathrm{I}_{\mathrm{TE}}$ \\
\hline Até 5 mil hab. & $\mathrm{I}_{\mathrm{TE}}<15,00 \% \rightarrow \mathrm{I}_{\mathrm{TE}}=0$ & $15,00 \%<\mathrm{I}_{\mathrm{TE}}<56,00 \% \rightarrow \mathrm{I}_{\mathrm{TE}}=1$ \\
De 5 a 20 mil hab. & $\mathrm{I}_{\mathrm{TE}}<16,50 \% \rightarrow \mathrm{I}_{\mathrm{TE}}=0$ & $16,50 \%<\mathrm{I}_{\mathrm{TE}}<63,75 \% \rightarrow \mathrm{I}_{\mathrm{TE}}=1$ \\
De 20 a 50 mil hab. & $\mathrm{I}_{\mathrm{TE}}<18,00 \% \rightarrow \mathrm{I}_{\mathrm{TE}}=0$ & $18,00 \%<\mathrm{I}_{\mathrm{TE}}<68,00 \% \rightarrow \mathrm{I}_{\mathrm{TE}}=1$ \\
De 50 a 100 mil hab. & $\mathrm{I}_{\mathrm{TE}}<26,00 \% \rightarrow \mathrm{I}_{\mathrm{TE}}=0$ & $26,00 \%<\mathrm{I}_{\mathrm{TE}}<72,25 \% \rightarrow \mathrm{I}_{\mathrm{TE}}=1$ \\
De 100 a 500 mil hab. & $\mathrm{I}_{\mathrm{TE}}<35,00 \% \rightarrow \mathrm{I}_{\mathrm{TE}}=0$ & $35,00 \%<\mathrm{I}_{\mathrm{TE}}<81,00 \% \rightarrow \mathrm{I}_{\mathrm{TE}}=1$ \\
$>$ 500 mil hab. & $\mathrm{I}_{\mathrm{TE}}<45,00 \% \rightarrow \mathrm{I}_{\mathrm{TE}}=0$ & $45,00 \%<\mathrm{I}_{\mathrm{TE}}<81,00 \% \rightarrow \mathrm{I}_{\mathrm{TE}}=1$ \\
\hline
\end{tabular}

Fonte: Adaptado CONESAN (1999).

\subsubsection{Indicador de drenagem urbana}

Para o Indicador de Drenagem Urbana ( $\left.I_{D U}\right)$, será avaliado nos bairros apenas a microdrenagem, dada a inexistência de macrodrenagem (canais naturais ou escoamento em fundos de vale). Segundo Mascaró (2005), recomenda-se para a estimativa da quantidade de bocas de lobo por área pavimentada, pelo menos 1 par de bocas de lobo a cada $400 \mathrm{~m}^{2}$ ou a cada $800 \mathrm{~m}^{2}$. Para o cálculo do I Du será considerado o critério de 1 par de bocas de lobo a cada $800 \mathrm{~m}^{2}$ de modo conservador. Dessa maneira, se o bairro possuir o número mínimo de bocas de lobo exigidas receberá pontuação 1 , caso contrário receberá pontuação 0 .

Neste trabalho, para a obtenção do comprimento das vias utilizou-se o Google Maps, um serviço de pesquisa e visualização de mapas e imagens. Para o cálculo da área de arruamentos e obtenção do quantitativo de bocas de lobo necessário, multiplicou-se a extensão das vias pela largura média de $7 \mathrm{~m}$. Para a quantidade atual de pares de bocas de lobo existente nos bairros em estudo, dispôs-se do estudo de concepção de gestão das águas pluviais desenvolvido pela Técnica de Engenharia Civil e Sanitária (TECISAN, 2014).

\subsubsection{Indicador de resíduos sólidos}

Segundo Menezes Filho e Rodrigues (2015), para o Indicador de Resíduos Sólidos $\left(I_{R S}\right)$ atribui-se pontuação de 0,0 a 1,0. Sendo 0,0 quando não há presença do subindicador e 1,0 quando há completa presença (Equação 8). Os subindicadores são a Coleta $\left(\mathrm{S}_{\mathrm{CL}}\right)$, Coleta seletiva $\left(\mathrm{S}_{\mathrm{CS}}\right)$, Reciclagem $\left(\mathrm{S}_{\mathrm{RC}}\right)$ e Aterro $\left(\mathrm{S}_{\mathrm{AT}}\right)$. Entretanto, o subindicador Coleta possui diferente pontuação, pois depende da frequência da 
coleta no local. Se a frequência é diária, é atribuído 1,0; ou 3 vezes na semana, 0,5; ou 2 vezes na semana, 0,25 ; ou 1 vez na semana, 0,0 .

$I_{R S}=\frac{S_{C L}+S_{C S}+S_{R C}+S_{A T}}{4}$

Em que: $I_{R S}$ - Indicador de Resíduos Sólidos; $S_{\mathrm{CL}}$ - Subindicador de Coleta; $\mathrm{S}_{\mathrm{CS}}$ - Subindicador de Coleta seletiva; $S_{R C}$ - Subindicador de Reciclagem; $S_{A T}$ Subindicador de Aterro.

\subsubsection{Indicador de controle de vetores}

O CONESAN (1999) apresenta o cálculo do Indicador de Controle de Vetores $\left(\mathrm{I}_{\mathrm{CV}}\right)$ como sendo a média aritmética entre o Indicador de Dengue $\left(\mathrm{I}_{\mathrm{VD}}\right)$, Indicador de Esquistossomose $\left(I_{V E}\right)$ e o Indicador de Leptospirose $\left(I_{V L}\right)$, exemplificado na Equação 9.

$$
\mathrm{I}_{\mathrm{CV}}=\frac{\mathrm{IVD}_{\mathrm{VD}}+\mathrm{I}_{\mathrm{VE}}+\mathrm{I}_{\mathrm{VL}}}{3}
$$

Em que: $I_{C V}$ - Indicador de Controle de Vetores; $I_{V D}$ - Indicador de Dengue; $I_{V E}$ - Indicador de Esquistossomose; I $I_{\mathrm{VL}}$ - Indicador de Leptospirose.

Os Indicadores de Dengue, Esquistossomose e Leptospirose são pontuados através da Tabela 5 .

Tabela 5 - Pontuação dos Indicadores de Dengue, Esquistossomose e Leptospirose.

\begin{tabular}{|c|c|c|c|}
\hline Dengue & $\begin{array}{c}\text { Critério } \\
\text { Esquistossomose }\end{array}$ & Leptospirose & Pontuação \\
\hline $\begin{array}{l}\text { Sem infestação por } \\
\text { Aedes Aegypti nos } \\
\text { últimos } 12 \text { meses }\end{array}$ & $\begin{array}{l}\text { Sem infestação por } \\
\text { esquistossomose } \\
\text { nos últimos } 5 \text { anos }\end{array}$ & $\begin{array}{c}\text { Sem enchentes e sem casos } \\
\text { de leptospirose nos últimos } 5 \\
\text { anos }\end{array}$ & 1,00 \\
\hline $\begin{array}{l}\text { Infestação por Aedes } \\
\text { Aegypti e sem } \\
\text { transmissão de dengue } \\
\text { nos últimos } 5 \text { anos }\end{array}$ & $\begin{array}{l}\text { Com incidência } \\
\text { anual }<1\end{array}$ & $\begin{array}{c}\text { Com enchentes e sem casos } \\
\text { de leptospirose nos últimos } 5 \\
\text { anos }\end{array}$ & 0,50 \\
\hline $\begin{array}{c}\text { Transmissão de } \\
\text { dengue nos últimos } 5 \\
\text { anos }\end{array}$ & $\begin{array}{l}\text { Com incidência } \\
\text { anual } \geq 1 \text { e }<5\end{array}$ & $\begin{array}{c}\text { Sem enchentes e com casos } \\
\text { de leptospirose nos últimos } 5 \\
\text { anos }\end{array}$ & 0,25 \\
\hline $\begin{array}{c}\text { Risco de ocorrência de } \\
\text { dengue hemorrágica }\end{array}$ & $\begin{array}{l}\text { Com incidência } \\
\quad \text { anual } \geq 5\end{array}$ & $\begin{array}{c}\text { Com enchentes e com casos } \\
\text { de leptospirose nos últimos } 5 \\
\text { anos }\end{array}$ & 0,00 \\
\hline
\end{tabular}

Fonte: Adaptado CONESAN (1999). 


\subsubsection{Indicador socioeconômico}

Segundo Oliveira (2014), o Indicador Socioeconômico ( $\left.I_{\mathrm{SE}}\right)$ pode ser calculado de acordo com a renda média mensal da população, seguindo a Equação 10.

$$
I_{S E}=\frac{v_{R}-v_{\text {MIN }}}{V_{M A ́ X}-v_{\text {MíN }}}
$$

Em que: $I_{S E}$ - Indicador Socioeconômico; $V_{R}-$ Valor da renda do local que será calculado; $\mathrm{V}_{\text {MíN }}$ - Valor mínimo da renda dos bairros a serem analisados; $\mathrm{V}_{\mathrm{MÁx}}$ Valor máximo da renda dos bairros a serem analisados.

A Tabela 6 apresenta as rendas médias mensais dos bairros a serem analisados.

Tabela 6 - Renda média dos bairros analisados

\begin{tabular}{cc}
\hline Bairro & $\begin{array}{c}\text { Renda média mensal em } \\
\mathbf{2 0 1 0}\end{array}$ \\
\hline Alto dos Caiçaras & $\mathrm{R} \$ 2.122,79$ \\
Cônego Getúlio & $\mathrm{R} \$ 1.510,71$ \\
Rosário & $\mathrm{R} \$ 1.029,57$ \\
Cerrado & $\mathrm{R} \$ 681,72$ \\
Cristo Redentor & $\mathrm{R} \$ 618,66$ \\
Nossa Senhora das Graças & $\mathrm{R} \$ 560,82$ \\
Alto da Colina & $\mathrm{R} \$ 408,66$ \\
Jardim Esperança & $\mathrm{R} \$ 381,00$ \\
\hline
\end{tabular}

Fonte: Adaptado do IBGE (2012).

Ressalta-se que os dados disponíveis para a realização da pesquisa referentes a renda média mensal são do ano de 2012; constituindo-se assim uma limitação, visto a possibilidade de alteração dos mesmos no cenário atual.

\section{RESULTADOS E DISCUSSÃo}

\subsection{Indicador de abastecimento de água}

Para o Indicador de Cobertura de Abastecimento de Água pode-se constatar o completo abastecimento em toda a área de Patos de Minas. No entanto, o Relatório de Fiscalização disposto pela Agência Reguladora de Serviços de Abastecimento de Água e Esgotamento Sanitário do Estado de Minas Gerais (ARSAE-MG, 2013) 
destacou nas entrevistas realizadas a intermitência no abastecimento no bairro Jardim Esperança, isto é o bairro de menor renda analisado.

O Indicador de Qualidade da Água Distribuída é de 99,7\% e foi disponibilizado pela COPASA, sendo analisado durante o período de setembro de 2018 até agosto de 2019. Segundo o Instituto Mineiro de Gestão de Águas (IGAM, 2018), no terceiro trimestre de 2017, esse Indicador na cidade era de 74,3\%, e em 2018 de 77,3\%. Além disso, a ARSAE no ano de 2013, apontava o parâmetro turbidez como fora dos padrões máximos permitidos.

Portanto, observa-se que houve uma evolução na distribuição de água na cidade, proporcionando melhores condições à população. O Indicador de Abastecimento de Água em toda a cidade de Patos de Minas-MG, calculado pela Equação 2, é de 99,9\%.

\subsection{Indicador de esgoto sanitário}

O Indicador de Cobertura em Coleta de Esgoto em Patos de Minas no ano de 2010, de acordo com o IBGE foi de $88,4 \%$, sendo o ICE igual a $100 \%$ conforme Tabela 2. Ainda segundo o IBGE (2012) o Indicador de Esgoto Tratado foi de apenas $10 \%$ no ano de 2010, sendo o $\mathrm{I}_{\mathrm{ET}}$ igual a zero conforme a Tabela 3. Até o presente momento, a cidade está estruturando a estação de tratamento de esgoto para que possa ampliar o atendimento. O resultado do Indicador de Esgoto Sanitário em toda a cidade de Patos de Minas, calculado pela Equação 5, é de 50,0\%.

\subsection{Indicador de drenagem urbana}

Dentre os bairros considerados, nenhum apresentou a quantidade de bocas de lobo necessária de acordo com a área; por conseguinte, o Indicador de Drenagem Urbana foi nulo (Tabela 7).

Os bairros Alto dos Caiçaras, Cônego Getúlio, Cristo Redentor, Alto da Colina e Jardim Esperança não possuem bocas de lobo. Devido à elevada inclinação longitudinal, os bairros Alto dos Caiçaras e Cônego Getúlio, com maior poder aquisitivo, não apresentam erosões. No entanto, nas vias nos bairros Alto da Colina e Jardim Esperança, classes de baixa renda, e que também apresentam ausência de dispositivos de microdrenagem, verificou-se diversas erosões (Figura 1). 
Figura 1 - Erosões nos bairros Jardim Esperança e Alto da Colina. Em (a), Jardim Esperança, e em (b), Alto da Colina.

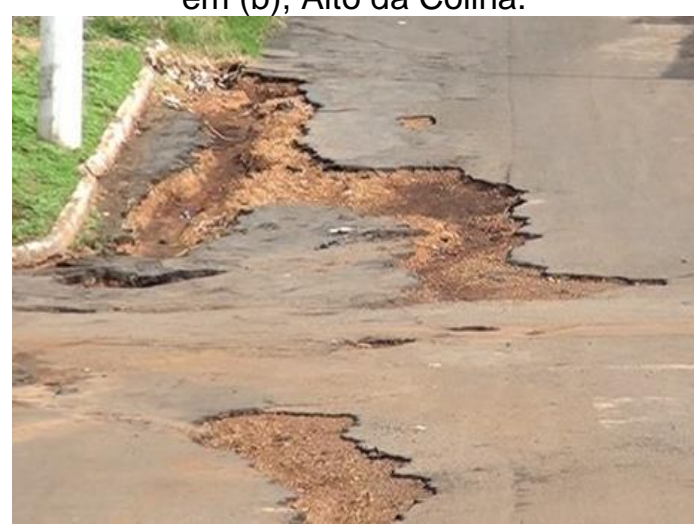

(a)

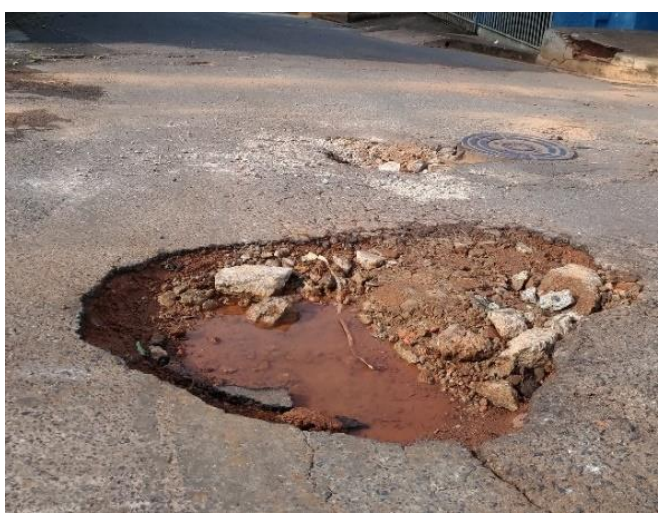

(b)

Fonte: Elaborado pelos autores.

O estudo de concepção de gestão das águas pluviais realizado em toda a cidade no ano de 2014, com o objetivo de obter o diagnóstico da gestão das águas pluviais, foi incisivo ao concluir a insuficiência de infraestruturas adequadas para captação e condução das águas pluviais, como bocas de lobo, galerias de águas pluviais e canais. Além disso, não há manutenção do sistema de micro e macrodrenagem intensificando as inundações durante o período chuvoso (TECISAN, 2014).

Tabela 7- Densidade de bocas de lobo.

\begin{tabular}{|c|c|c|c|c|c|}
\hline Bairro & $\begin{array}{c}\text { Área de } \\
\text { arruamento } \\
\left(\mathrm{m}^{2}\right)\end{array}$ & $\begin{array}{c}\text { Quantidade de } \\
\text { bocas de lobo } \\
\text { recomendadas } \\
\text { (pares) }\end{array}$ & $\begin{array}{c}\text { Quantidade } \\
\text { atual (pares) } \\
\text { (TECISAN,204) }\end{array}$ & Déficit & $\begin{array}{l}\text { Déficit } \\
(\%)\end{array}$ \\
\hline $\begin{array}{l}\text { Alto dos } \\
\text { Caiçaras }\end{array}$ & $67.296,5$ & 118 & 0 & 118 & $100 \%$ \\
\hline $\begin{array}{l}\text { Cônego } \\
\text { Getúlio }\end{array}$ & $46.140,4$ & 81 & 0 & 81 & $100 \%$ \\
\hline $\begin{array}{l}\text { Rosário } \\
\text { Cerrado }\end{array}$ & $\begin{array}{l}33.225,5 \\
18.459,0\end{array}$ & $\begin{array}{l}58 \\
33\end{array}$ & $\begin{array}{l}6 \\
2\end{array}$ & $\begin{array}{l}52 \\
31\end{array}$ & $\begin{array}{l}90 \% \\
94 \%\end{array}$ \\
\hline $\begin{array}{l}\text { Cristo } \\
\text { Redentor } \\
\text { Nossa }\end{array}$ & $81.319,0$ & 143 & 0 & 143 & $100 \%$ \\
\hline $\begin{array}{c}\text { Senhora das } \\
\text { Graças }\end{array}$ & $47.516,0$ & 83 & 5 & 78 & $94 \%$ \\
\hline \multirow{2}{*}{$\begin{array}{c}\text { Alto da Colina } \\
\text { Jardim } \\
\text { Esperança }\end{array}$} & $26.872,5$ & 47 & 0 & 47 & \multirow{2}{*}{$\begin{array}{l}100 \% \\
100 \%\end{array}$} \\
\hline & $59.008,5$ & 104 & 0 & 104 & \\
\hline
\end{tabular}

Fonte: Elaborado pelos autores. 


\subsection{Indicador de resíduos sólidos}

Segundo o IBGE (2012), 92,3\% dos resíduos produzidos eram recolhidos na cidade, mas a coleta não compreendia a zona rural pertencente ao município. Além disso, a estimativa é de 113 t/dia de lixo recolhidos. De acordo com o SNIS (2017), a disposição final dos resíduos sólidos em Patos de Minas é feita no aterro sanitário. No entanto, há apenas o tratamento do chorume interno.

Em relação à reciclagem, a cidade contava com a Associação Patense de Reciclagem (Apare), que iniciou as atividades em 2003 e realizava a coleta de 42 toneladas de recicláveis mensalmente. Todavia, as tentativas de implementação de autogestão, regularização e aumento das parcerias firmadas (SOUZA, 2014), não foram suficientes para evitar o encerramento das atividades em 2019. Dessa forma, a cidade necessitará de investimentos para retomar a coleta de recicláveis e iniciar a coleta seletiva.

Dentre os bairros analisados, apenas o Rosário possui coleta diária, os demais possuem coleta três vezes na semana. Portanto, pode-se constatar que independentemente da renda, o serviço oferecido alcança a todos os bairros estudados.

Utilizando a média aritmética dos indicadores de coleta seletiva, reciclagem e frequência de coleta pode-se obter os $I_{R S}$ (Figura 2).

Figura 2 - Resumo Indicador de Resíduos Sólidos

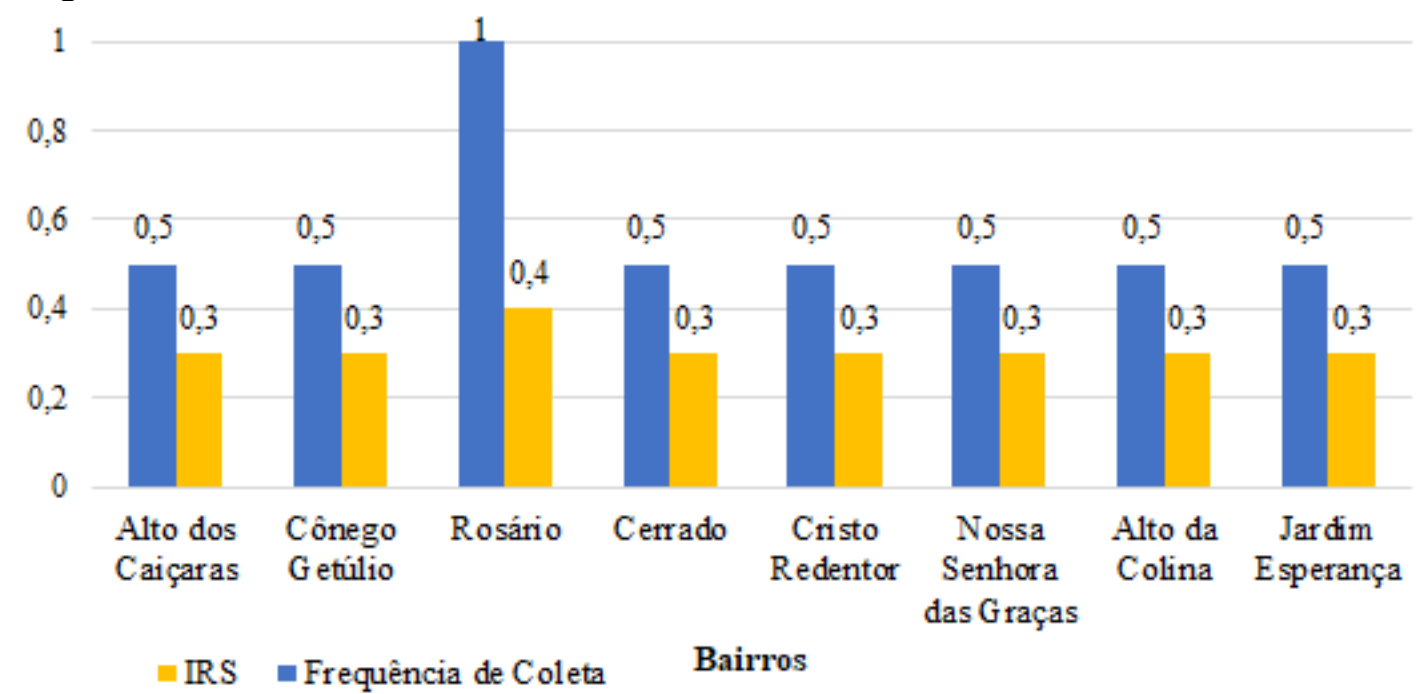

Fonte: Elaborado pelos autores. 
Vale ressaltar a importância da participação da população ao manusear 0 resíduo produzido, por exemplo, separar o lixo orgânico do reciclável. Isso facilita o trabalho para os catadores de recicláveis e evita o descarte incorreto do que pode ser aproveitado. No entanto, a ABRELPE (2017) mostra que 75\% dos municípios brasileiros não possuem a iniciativa de separar o lixo.

Nos bairros em estudo há focos de resíduos que não possuem destinação correta. No entanto, em bairros de alta renda a quantidade é inferior, em comparação aos bairros de baixa renda (Figura 3). Além disso, observa-se a não separação dos resíduos, a dependência entre a renda e a atitude por parte dos cidadãos em mudar esse cenário.

Figura 3 - Descarte não ideal nos bairros Alto dos Caiçaras e Alto da Colina. Em (a), Alto dos Caiçaras, e em (b), Alto da Colina

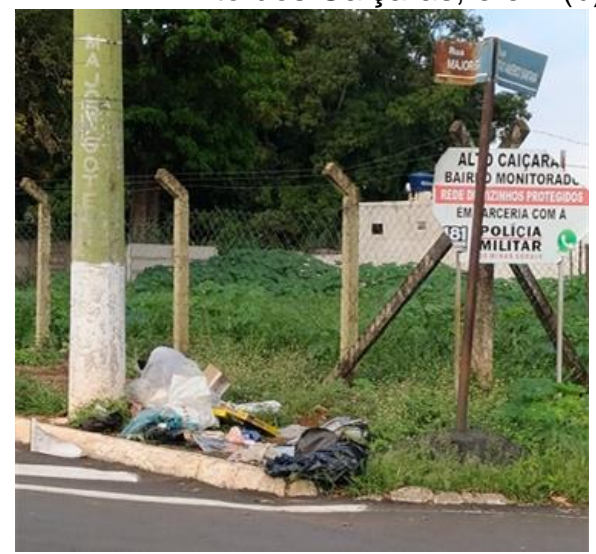

(a)

Fonte: Elaborado pelos autores.

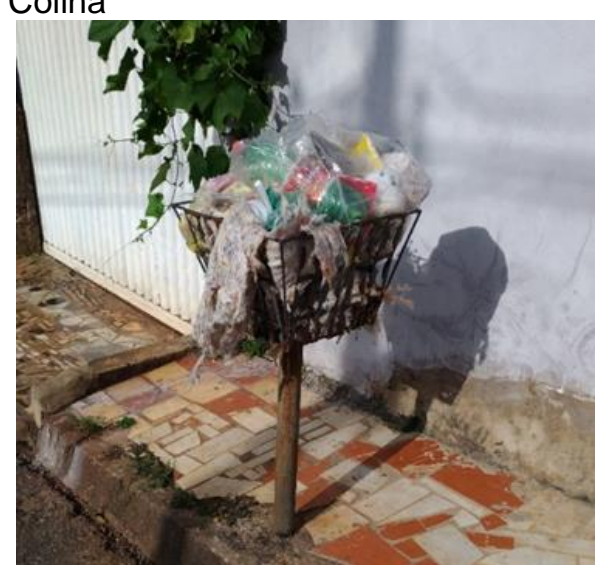

(b)

\subsection{Indicador de controle de vetores}

Quanto ao Indicador de Controle de Vetores, não foram verificados casos de esquistossomose e leptospirose. Já em relação a dengue, apenas os bairros de maior poder aquisitivo, Alto dos Caiçaras e Cônego Getúlio, não tiveram infestação de dengue no último censo disponibilizado pela Prefeitura Municipal (2018). Esse evento pode ser justificado devido a menor quantidade de lixo sem a destinação correta em comparação aos outros bairros. Por exemplo, no Alto da Colina, constatou-se lixo de diversas origens, incluindo ossos de animais de grande porte (Figura 4). 


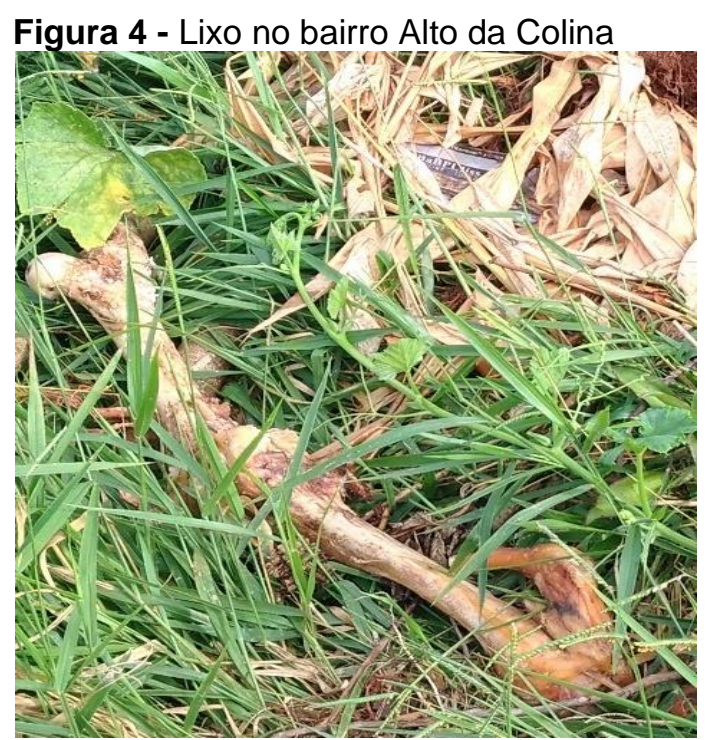

Fonte: Elaborado pelos autores.

Sobral (2019) em seu estudo identificou que locais onde há maior coleta de resíduos são menos sujeitos a casos de dengue. Logo, deve-se buscar alternativas que proporcionem otimização nos processos de limpeza da cidade. Na Figura 5, apresentam-se os valores obtidos para o Indicador de Controle de Vetores.

Figura 5 - Gráfico do Indicador de Dengue ( $\left.I_{V D}\right)$.

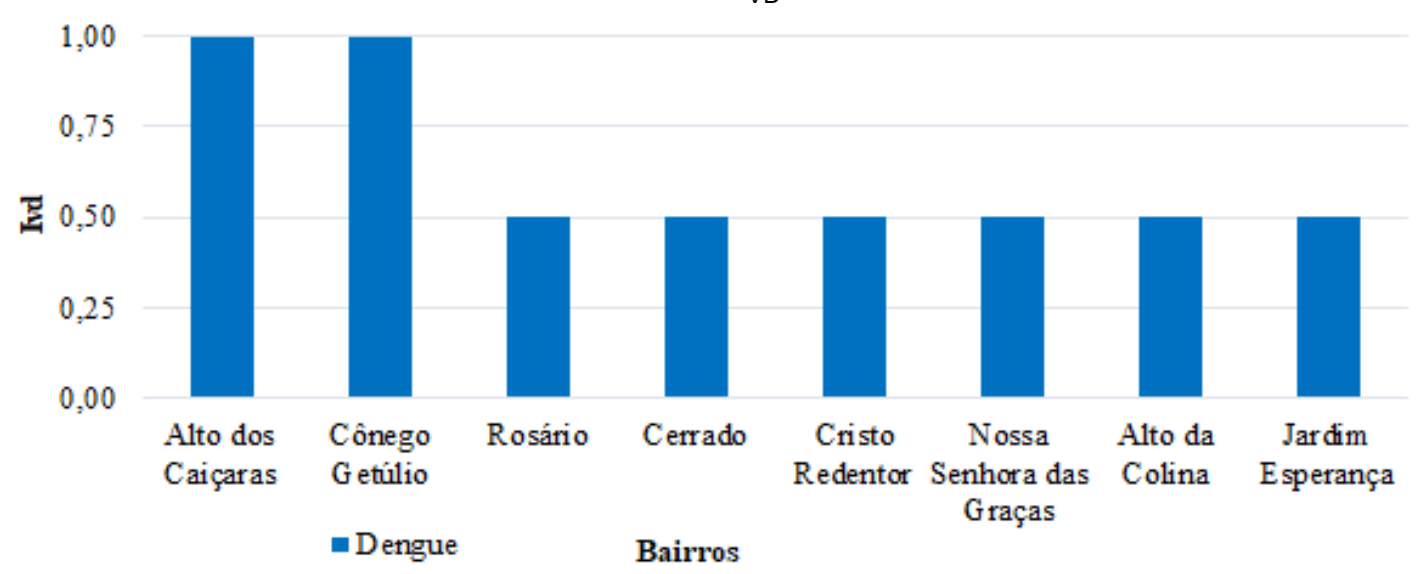

Fonte: Elaborado pelos autores.

\subsection{Indicador socioeconômico}

Para o Indicador Socioeconômico é possível observar o comprometimento dos órgãos gestores da cidade nos bairros de maior renda, afinal, é esperado que, com a maior arrecadação, o município realize os serviços necessários. Caso contrário, sem as manutenções necessárias, haverá desvalorização do local. 
Na Figura 6 apresentam-se os Indicadores de Salubridade Ambiental (ISA) e o Socioeconômico (ISE) para os bairros analisados. Apenas o bairro Alto dos Caiçaras foi classificado como de média salubridade e os demais como de baixa salubridade.

Figura 6 - Gráfico resumo do Indicador de Salubridade Ambiental (ISA) e Indicador

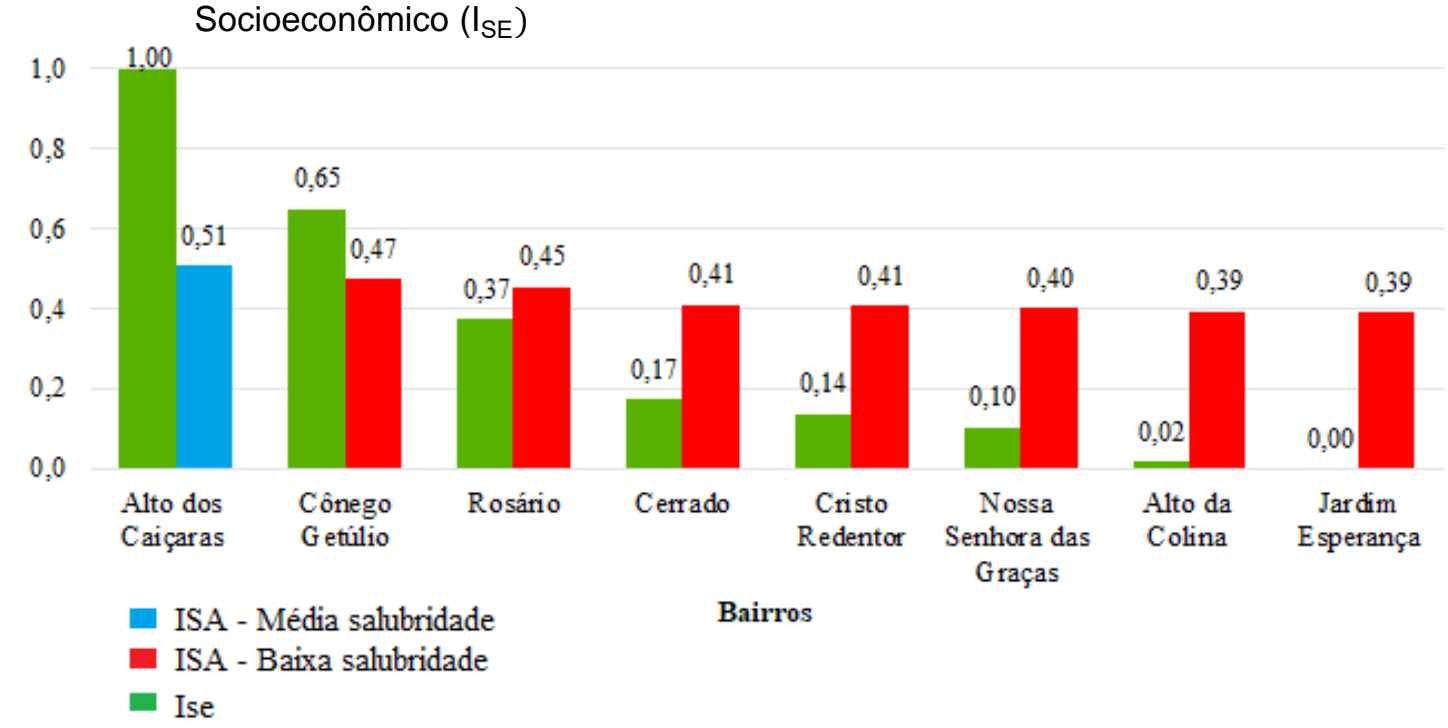

Fonte: Elaborado pelos autores.

Ressalta-se que os resultados apresentados neste trabalho denotam o descaso do poder público municipal quanto aos serviços prestados relacionados ao saneamento ambiental. Todos os bairros em análise foram criados nos anos 90 e não se configuram como ocupações recentes. São bairros distantes do centro, criados em alguns casos, pela necessidade da população por moradias seguras ao se afastar das inundações ribeirinhas do Rio Paranaíba. Tal qual apresenta DIAS (2005) em seu trabalho intitulado Tensões urbanas - trajetórias e vivências de moradores do Bairro Alto da Colina na luta pelo espaço urbano (Patos de Minas 1980-2004), ao retratar a luta dos moradores do bairro supracitado por uma vida mais digna, decorrente dos alagamentos do Bairro Vila Operária de onde partiram:

Muros são erguidos, flores germinam pelas praças e a cidade vai seguindo seu fluxo. Entre lotes baldios, lixos fétidos, carcaças de animais em estado de putrefação no solo urbano, caminham vidas, passam longas horas buscando trabalho, catando pedaços de sobrevivência pelos lixos, recolhendo papelões, insistindo em sobreviver, pertencer a esse palco urbano (DIAS, 2005, p.60). 


\section{CONCLUSÕES}

O presente trabalho avaliou a salubridade ambiental por meio da aplicação do ISA em bairros de classes econômicas distintas na cidade de Patos de Minas-MG. A partir do cálculo dos Indicadores, pôde-se constatar que apenas os Indicadores de Abastecimento de Água e Controle de Vetores foram considerados satisfatórios em todos os bairros analisados. Para esses dois componentes, não houve relação entre a renda do bairro e a presença dos serviços de saneamento. Contudo, no bairro Jardim Esperança, de menor renda entre os demais, verificou-se relatos de intermitência no abastecimento de água, apontando a necessidade de melhorias no sistema por parte da companhia de saneamento.

Em relação aos Indicadores de Esgoto Sanitário, Resíduos Sólidos e Drenagem Urbana, observou-se uma deficiência em todos os bairros estudados, independente da renda econômica. Ressalta-se que para o Indicador de Drenagem urbana, expresso pela presença de microdrenagem (bocas de lobo), obteve-se valor nulo em todos os bairros, indicando a carência do serviço de manejo de águas pluviais.

Quanto ao Indicador de Resíduos Sólidos, para o bairro Alto da Colina, onde fora observado diversos focos de lixo acumulado, verifica-se a necessidade da ampliação da frequência de coleta de lixo, bem como, a promoção de campanhas de conscientização sobre o descarte inadequado de resíduos e seus impactos sobre o meio ambiente.

O Indicador Socioeconômico propiciou para o bairro Alto dos Caiçaras, de maior renda média mensal, a classificação de média salubridade para o ISA. A alta arrecadação de impostos e a maior conscientização por parte da população residente neste bairro, em buscar soluções que garantam que os resíduos possuam destinação correta, foram determinantes para tal classificação.

Conclui-se, portanto, que bairros de classes econômicas distintas apresentam diferentes indicadores de salubridade ambiental. Tal evidência, pode auxiliar aos gestores municipais ou concessionárias dos serviços de saneamento ambiental, na melhor tomada de decisões quanto à políticas públicas e investimentos, sobretudo nos bairros de menor renda média mensal. 


\section{AGRADECIMENTOS}

Os autores agradecem à Prefeitura Municipal de Patos de Minas-MG, ao escritório do IBGE e também à COPASA pelas informações e dados necessários ao desenvolvimento deste trabalho.

\section{REFERÊNCIAS}

ABRELPE. Associação Brasileira Empresas de Limpeza Pública e Resíduos Especiais. Panorama dos resíduos sólidos no Brasil. 15.ed. São Paulo, 2017. 73 p.

ABRELPE. Associação Brasileira Empresas de Limpeza Pública e Resíduos Especiais. Panorama dos resíduos sólidos no Brasil. 16.ed. São Paulo, 2019. 64 p.

ARSAE. Agência reguladora de serviços de abastecimento de água e esgotamento sanitário do estado de Minas Gerais. Fiscalização do sistema de esgotamento sanitário da sede do Município de Patos de Minas. Belo Horizonte, 2013.

BRASIL. Lei n 11445, de 5 de janeiro de 2007. Saneamento básico. Brasília, 2007. Disponível em:

http://www.diariodasleis.com.br/busca/exibelink.php? numlink=1-98-24-2007-01-05-11445.

Acesso em: 13 mar. 2020

BROWN, C.; NEVES-SILVA, P.; HELLER, L. The human right to water and sanitation: a new perspective for public policies. Ciência \& Saúde Coletiva, v. 21, n. 3, p. 661-670, 2016. https://doi.org/10.1590/1413-81232015213.20142015

CADERNO DE GRADUAÇÃO: impactos ambientais referentes à não coleta de lixo e reciclagem. Maceió, 2015. ISSN 2357-9919.

CONSELHO ESTADUAL DE SANEAMENTO (CONESAN). ISA - Indicador de salubridade ambiental: manual básico. São Paulo, 1999.

DIAS, M. C. Índice de salubridade ambiental em áreas de ocupação espontânea: estudo em Salvador, Bahia. 2003. 171f. Dissertação (Mestrado em Engenharia Ambiental Urbana)Universidade Federal da Bahia, Salvador.

DIAS, C. C. Tensões urbanas: trajetórias e vivências de moradores do Bairro Alto da Colina na luta pelo espaço urbano (Patos de Minas 1980-2004). 2005. 100f. Dissertação (Programa de Mestrado em História) - Universidade Federal de Uberlândia, Minas Gerais, 2005.

IBGE, Instituto Brasileiro de Geografia e Estatística. Censo Brasileiro de 2010. Rio de Janeiro: IBGE, 2012.

INSTITUTO TRATA BRASIL. Ranking do saneamento Instituto Trata Brasil 2020 (SNIS 2018). São Paulo, 2020. Disponível em:

http://www.tratabrasil.org.br/images/estudos/itb/ranking 2020/Relatorio Ranking Trata Bras il 2020 Julho .pdf . Acesso em: 17 jul. 2020.

IGAM. Instituto Mineiro de Gestão das Águas. Monitoramento da qualidade das águas 
superficiais no estado de Minas Gerais. Relatório Trimestral. Belo Horizonte, 2018.

HELLER, L; PÁDUA, V. L. Abastecimento de água para consumo humano. Belo Horizonte: Editora UFMG, 2006. 859 p.

LIMA, A. S. C.; ARRUDA, P. N.; SCALIZE, P.S. Indicador de salubridade ambiental em 21 municípios do estado de Goiás com serviços públicos de saneamento básico operados pelas prefeituras. Engenharia Sanitária e Ambiental, 2019.https://doi.org/10.1590/s1413$\underline{41522019188336 .}$.

LINS, A. F.; MORAES, A. R. Determinação do Índice de Salubridade Ambiental no município de Guaíra-PR, Brasil. In: CONGRESSO BRASILEIRO DE GESTÃO AMBIENTAL, 8., 2017. Campo Grande. [Anais...]. Campo Grande, 2017. Disponível em: http://www.ibeas.org.br/congresso/Trabalhos2017/IX-003.pdf

LISBOA, S. S.; HELLER, L.; SILVEIRA, R. B. Desafios do planejamento municipal de saneamento básico em municípios de pequeno porte: a percepção dos gestores. Eng. Sanit. Ambient., Rio de Janeiro, v. 18, n. 4, p. 341-348, Dec. 2013. https://doi.org/10.1590/S1413-41522013000400006.

MENEZES FILHO, F. C. M; RODRIGUES, A. L. M. O modelo ISA como indicador de salubridade ambiental para o município de Rio Paranaíba/MG. In: XXI SIMPÓSIO BRASILEIRO DE RECURSOS HÍDRICOS, 21., 2015. [Anais...]. Brasília, DF, 2015.

MASCARÓ, J. L. Loteamentos urbanos. Porto Alegre: Masquatro, 2005. 210 p. OLIVEIRA, F. S. O modelo ISA utilizado no diagnóstico da salubridade ambiental nos bairros do município de Juiz de Fora-MG. Trabalho Final de Curso. (Graduação) Faculdade de Engenharia da UFJF. Juiz de Fora, 2014.

ONU. Organização das Nações Unidas. Assembléia Geral da ONU reconhece saneamento como direito humano distinto do direito à água potável. 2015. Disponível em: https://nacoesunidas.org/assembleia-geral-da-onu-reconhece-saneamento-como-direitohumano-distinto-do-direito-a-agua-potavel/. Acesso em: 13 mar. 2020.

SANTAELLA, S. T. et al. Resíduos sólidos e a atual política ambiental brasileira. Fortaleza: UFC / LABOMAR / NAVE, 2014. v. 7. 232 p.

SALLA, M. R. et al. Relação entre saneamento básico e saúde pública em Bissau, GuinéBissau. Saúde soc., São Paulo, v. 28, n. 4, p. 284-296, 2019. https://doi.org/10.1590/S0104-12902019180705.

SILVA JÚNIOR, L. G. da. Características das precipitações pluviométricas em Patos de Minas - MG: análise da intensidade-duração-frequência das chuvas. 56 p. Monografia (Graduação em Geografia) - Universidade Federal de Uberlândia. Uberlândia-MG, 2015.

SNIS. Diagnóstico do manejo de resíduos sólidos urbanos. 2017 Disponível em: http://www.snis.gov.br/diagnostico-anual-residuos-solidos/diagnostico-rs-2017. Acesso em: 17 jul. 2020.

SNIS. Diagnóstico de drenagem e manejo das águas pluviais urbanas. 2018 Disponível em: http://www.snis.gov.br/downloads/diagnosticos/ap/2018/Diagnostico AP2018.pdf. Acesso em: 16 mar. 2020.

SOBRAL, M. F. F.; SOBRAL, A. I. G. P. Casos de dengue e coleta de lixo urbano: um 
estudo na Cidade do Recife, Brasil. Ciência \& Saúde Coletiva [online], v. 24, n. 3, p. 1075-1082, 2019. https://doi.org/10.1590/1413-81232018243.10702017.

SOUZA, T. S. Organização e estruturação da associação patense de reciclagem visando a coleta seletiva do município de Patos de Minas - MG: estudo de caso. Dissertação (Mestrado) - Universidade Federal do Tocantins Programa Mestrado Profissional em Engenharia Ambiental Saneamento, 2014.

UHR, J. G. Z. et al. Relação entre saneamento básico no Brasil e saúde da população sob a ótica das internações hospitalares por doenças de veiculação hídrica. RACEF - Revista de Administração, Contabilidade e Economia da Fundace. v. 7, n. 2, p. 01-16, 2016. http://dx.doi.org/10.13059/racef.v7i2.104.

TÉCNICA DE ENGENHARIA CIVIL E SANITÁRIA (TECISAN). Manejo de águas pluviais de drenagem urbana do município de Patos de Minas-MG: estudo de concepção de gestão das águas pluviais. Belo Horizonte, 2014.

TEIXEIRA, D.A. Construção e determinação do indicador de salubridade ambiental (ISA/OP) para as áreas urbanas do município de Ouro Preto, MG. 2017. $154 \mathrm{f}$.

Dissertação (Mestrado em Engenharia Ambiental) - Núcleo de Pesquisas e Pós-Graduação em Recursos Hídricos, Universidade Federal de Ouro Preto. Ouro Preto, 2017.

TEIXEIRA, D.A; FILHO, J.F.P; SANTIAGO, A.F. Indicador de salubridade ambiental: variações da formulação e usos do indicador no Brasil. Ouro Preto. 2018. https://doi.org/10.1590/s1413-41522018170866.

TRIGUEIRO, A. W. B. et al. A influência dos fatores socioeconômicos sobre o saneamento básico na população do município de Araruna-PB. In: CONGRESSO INTERNACIONAL DA DIVERSIDADE DO SEMIÁRIDO - CONIDIS, 1., 2016. [Anais....]. 2016. 